\title{
Characterization of Brazilian Syrah winter wines at bottling and after ageing
}

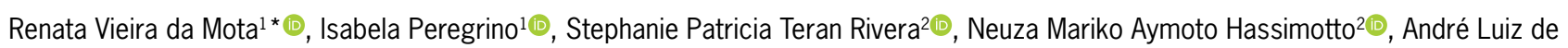
Souza $^{3}$, Claudia Rita de Souza ${ }^{10}$

${ }^{1}$ Empresa de Pesquisa Agropecuária de Minas Gerais/Núcleo Tecnológico Uva e Vinho. Av. Santa Cruz, 500, C.P. $33-$ 37780-000 - Caldas, MG - Brasil.

ZUniversidade de São Paulo/Faculdade de Ciências Farmacêuticas/Centro de Pesquisas em Alimentos - Depto. de Alimentos e Nutrição Experimental, Av. Prof. Lineu Prestes, 580 - Bloco 14 - 05508-000 - São Paulo, SP Brasil.

3Universidade Federal de Alfenas - Depto. de Química, Rod. José Aurélio Vilela, 11999 - 37715-400 - Poços de Caldas, MG - Brasil.

*Corresponding author <rvmota@epamig.br>

Edited by: Luís Guilherme de Lima Ferreira Guido

Received August 29, 2019

Accepted December 27, 2019
ABSTRACT: Double pruning extended the harvest season of wine grape (Vitis vinifera L.) to dry winter, enabling production of high quality wines in the southeastern Brazil. Winter harvest allows grapes to fulfill not only technological maturation, but also phenolic ripeness. Winter wines from Syrah grapes harvested from eight vineyards in southeastern Brazil during three harvests were analyzed for their chemical and aromatic composition after bottling and after ageing for 20, 30, and 42 months in bottle. Winter wines have high content of total phenolic compounds, which remained almost constant through ageing, as well as color intensity. Malvidin 3-0-glucoside stood out among anthocyanins, remaining 5-10\% after 39 months of ageing. Moreover, malvidin 3-0-glucoside-pyruvic acid was the main pyranoanthocyanin identified in winter wine. Polymerized pigments index ranged from $54 \%$ at bottling to $80 \%$ after 42 months of ageing. Young winter wines are rich in ester and monoterpene, as well as alcoholic volatile compounds responsible for ethereal, fruity, flowery, fresh and sweet aromas. Aged winter wines showed higher contents of furfural, geranyl ethyl ether, isoamyl decanoate, $\alpha$-muurolene and $\alpha$-calacorene, contributing to sweet, fruity and woody aromas. Syrah winter wines are characterized by high content of phenolic compounds and color stability, and keep good sensorial characteristics after ageing in bottle.

Keywords: double-pruning, composition, color, phenolics, aroma

\section{Introduction}

The use of double pruning enables to harvest grapes under climatic conditions that favor grape ripening (Favero et al., 2011; Palliotti et al., 2017; Toda et al., 2019). In southeastern Brazil, this technique allowed vineyard dissemination for high quality fine wine production (Favero et al., 2011).

Wines elaborated from grapes harvested at winter season under low water availability and high thermal amplitude have higher alcoholic and phenols content as well as color intensity than summer wines (Mota et al., 2009). Similar results were obtained with controlled water deficits in 'Cabernet sauvignon' vines (CáceresMella et al., 2018). The authors observed that wines from less irrigated vines exhibited high levels of total phenols, anthocyanins and chroma and therefore showed higher color intensity and sensorial perception of more fullness. Phenolic compounds are the main component of color and mouthfeel in wine, also affecting its ageing ability. Double pruning favors phenolic maturation mainly by high thermal amplitude and low rainfall during autumnwinter season (Favero et al., 2011).

Besides color, aroma is a wine characteristic immediately observed and highly appreciated by consumers. The presence of compounds, such as alcohols, acids, aldehydes, lactones, benzenes, esters, ketones, terpenes, originating from grapes or formed during the fermentation process and ageing, are responsible for the aroma profile of the wine (Vidal and Segurel, 2005; Belda et al., 2017).

Among the cultivars tested under double-pruning management in the southeastern Brazil (Mota et al.,
2010, 2011), Syrah vines showed the best adaptation to this management. Winter Syrah wines have been well accepted by consumers, which increased the interest in management of this vineyard in Brazil (Salettes, 2016); however, there is little information on the composition of these wines and the effect of ageing.

As far as the authors know, there is no information in the literature about aromatic profile of Syrah wines from winter harvest. Therefore, this work investigated the volatile aroma compounds in Syrah wines from winter harvest and the effect of bottle ageing in the composition of these wines.

\section{Materials and Methods}

\section{Wine samples}

Wines were elaborated from grapes harvested in the 2013, 2014 and 2015 seasons from non-irrigated commercial vineyards in Indaiatuba, SP $\left(23^{\circ} 05^{\prime} \mathrm{S}\right.$, $47^{\circ} 13^{\prime} \mathrm{W}$, altitude of $701 \mathrm{~m}$ ), Santo Antônio do Amparo, MG $\left(20^{\circ} 95^{\prime} \mathrm{S}_{1} 4^{\circ} 4^{\circ} 91^{\prime} \mathrm{W}_{i,}\right.$ altitude of $\left.990 \mathrm{~m}\right)$, São Bento do Sapucaí, SP $\left(22^{\circ} 41^{\prime} \mathrm{S}, 45^{\circ} 43^{\prime} \mathrm{W}\right.$, altitude of 886 m), São Sebastião do Paraíso, MG $\left(20^{\circ} 54^{\prime} \mathrm{S}, 47^{\circ} 06^{\prime} \mathrm{W}\right.$, altitude of $860 \mathrm{~m})$, Três Pontas, MG $\left(21^{\circ} 12^{\prime} \mathrm{S}, 4^{\circ} 35^{\prime}\right.$ $\mathrm{W}$, altitude of $881 \mathrm{~m})$, Vargem, SP $\left(22^{\circ} 53^{\prime} \mathrm{S}, 46^{\circ} 24^{\prime} \mathrm{W}\right.$, altitude of $845 \mathrm{~m})$, Itobi, SP $\left(21^{\circ} 42^{\prime} \mathrm{S}, 46^{\circ} 55^{\prime} \mathrm{W}\right.$, altitude of $840 \mathrm{~m}$ ) and Três Corações, MG (21 $1^{\circ} 36^{\prime} \mathrm{S}, 4^{\circ} 07^{\prime} \mathrm{W}$, altitude of $865 \mathrm{~m}$ ) in the southeastern region of Brazil. All vineyards were over 6 years old of 'Syrah' clone 174 ENTAV-INRA, grafted onto 1103 Paulsen, at vertical shoot position trellis managed under double pruning. The first pruning to induce the vegetative cycle was carried out in September and all bunches at bunch closure stage 
were removed to avoid harvest in the summer season. In January of the subsequent year, the lignified shoots were pruned to allow the productive cycle during the autumn-winter season (Favero et al., 2011).

Grapes were harvested on average with $23.1^{\circ} \mathrm{Brix}$, $\mathrm{pH} 3.61$ and total acidity $5.92 \mathrm{~g} \mathrm{~L}^{-1}$ tartaric acid. Not all vineyards were represented in the three seasons due to environmental damages or erroneous management that compromised vine production.

Harvested bunches were stored at $4{ }^{\circ} \mathrm{C}$ for $24 \mathrm{~h}$ at the winery. For each region, $200 \mathrm{~kg}$ of grape clusters were destemmed, crushed and placed in $200 \mathrm{~L}$ inox fermentation tanks. The musts were inoculated with rehydrated wine yeast Saccharomyces cerevisiae $\times S$. kudriavzevii and added with $80 \mathrm{mg} \mathrm{SO}_{2} \mathrm{~kg}^{-1}$.

Wine density was determined daily during alcoholic fermentation performed at $21{ }^{\circ} \mathrm{C}$. When the density reached approximately $990 \mathrm{~g} \mathrm{~L}^{-1}$, wines were racked to $100 \mathrm{~L}$ stainless steel tanks. Malolactic fermentation was carried out with native bacteria flora at $21^{\circ} \mathrm{C}$ and the presence of malic acid was routinely followed by the paper chromatography method (Amerine and Ough, 1980). When malic acid was not detected by paper chromatography, wines were carefully racked to avoid lees, added with $35 \mathrm{mg} \mathrm{SO} \mathrm{L}^{-1}$ and kept at $3{ }^{\circ} \mathrm{C}$ for $15 \mathrm{~d}$ to allow tartaric stabilization. The wines were bottled in $750 \mathrm{~mL}$ green glass bottles closed with natural cork and allowed to age in lying position at $15^{\circ} \mathrm{C}$ in a dark cell for 20,30 or 42 months.

\section{Wine composition}

The physicochemical analyses consisted of alcohol, fixed acidity $\left(\mathrm{g} \mathrm{L}^{-1}\right.$ tartaric acid), $\mathrm{pH}$, residual sugars (g $\left.\mathrm{L}^{-1}\right)$ and ashes $\left(\mathrm{g} \mathrm{L}^{-1}\right)$ (Amerine and Ough, 1980). Color intensity (CI) $\left(\mathrm{A}_{420}+\mathrm{A}_{520}+\mathrm{A}_{620}\right)$, color hue $\left(\mathrm{A}_{420} / \mathrm{A}_{520}\right)$, color composition (the contribution of each color component in the overall color expressed by the general equation OD $\mathrm{n}(\%)=\mathrm{OD} \mathrm{n} / \mathrm{CI} \times 100)$ and polymerized pigments were evaluated by spectrophotometry (Ribéreau-Gayon et al., 2006; Harbertson and Spayd, 2006). Total flavanoid content was evaluated by Bate-Smith reaction (RibéreauGayon et al., 2006). Anthocyanins and phenolics were measured by the $\mathrm{pH}$ differential method (Giusti and Wrosltad, 2000) and Folin-Ciocalteu method (Amerine and Ough, 1980), respectively. Each sample was analyzed in triplicate at bottling and after ageing.

\section{Monomeric Anthocyanin extraction}

Anthocyanins and polymeric anthocyanins identification were performed in Syrah winter wines produced in São Bento, Três Pontas, Indaiatuba, Santo Antônio do Amparo and São Sebastião do Paraíso at bottling and after 19 and 36 months of ageing.

Identification and quantification of monomeric anthocyanins were performed by the HPLC-DAD and LC-ESI-MS/MS analyses. Wines samples $(5 \mathrm{~mL})$ were concentrated under vacuum at $40{ }^{\circ} \mathrm{C}$ on a rotary evaporator to remove the alcohol, and filled up to $10 \mathrm{~mL}$ with ultrapure water prior to application to a solid-phase extraction polyamide column (1 g) previously conditioned with methanol and ultrapure water. The de-alcoholized sample $(2 \mathrm{~mL})$ was loaded into the column and washed with ultrapure water and eluted with $0.3 \% \mathrm{HCl}$ in methanol. The eluates were completely dried using a rotary evaporator under vacuum at $40{ }^{\circ} \mathrm{C}$, resuspended in $5 \%$ acetic acid in methanol and filtered through a 0.45 $\mu \mathrm{m}$ PTFE filter. For the polymeric anthocyanin analysis, wine was filtered through a $0.45 \mu \mathrm{m}$ PTFE and injected directly.

\section{LC-ESI-MS/MS and HPLC-DAD conditions}

Anthocyanins were identified by LC-ESI-MS/MS using a Prominence Liquid Chromatograph attached to an ion trap Esquires-LC mass spectrometer with an electrospray ionization (ESI) interface. We used a 5- $\mu$ Prodigy ODS3 column $(4.60 \times 250 \mathrm{~mm})$ with a flow rate of $1 \mathrm{~mL} \mathrm{m^{-1 }}$ at $25{ }^{\circ} \mathrm{C}$. The mobile phase consisted of solvent $\mathrm{A}$, water ultrapure, formic acid and acetonitrile $(95: 1: 3, \mathrm{v} / \mathrm{v} / \mathrm{v})$ and solvent $\mathrm{B}$, water, formic acid and acetonitrile $(48: 1: 51, \mathrm{v} / \mathrm{v} / \mathrm{v})$. Anthocyanins were detected at $525 \mathrm{~nm}$ (Teixeira et al., 2015). For application to the mass spectrometer after DAD detection, the flow rate was reduced to $0.2 \mathrm{~mL} \mathrm{~min}^{-1}$ Mass spectrometer operated with collision energies of $-3500 \mathrm{~V}$ and $\mathrm{N}_{2}$ like dry gas with ESI in the positive mode using a full scan from $\mathrm{m} / z 100$ to 1500 . Compounds were identified according to comparison with the retention time of authentic standards when possible, as well as by absorption spectrum similarity, mass spectral characteristics and by comparison with the literature data. The calibration curve was performed by injecting the standards malvidin 3-O-glucoside, peonidin 3-O-glucoside, petunidin 3-O-glucoside, delphinidin 3-O-glucoside three times at five different concentrations. The acylated form of anthocyanins with coumaroyl and acetyl groups were quantified using the calibration curve of their respective O-glucoside form. The results were expressed as mg $100 \mathrm{~mL}^{-1}$.

\section{Pyranoanthocyanins analysis by LC-qTOF-MS/MS and HPLC-DAD conditions}

The identification of the polymeric anthocyanins was performed using a Prominence Liquid Chromatograph linked to a qTOF mass spectrometer Compact model. The LC condition was reverse phase Luna $3 \mu \mathrm{C} 18(150 \times 3.0$ $\mathrm{mm})$ at $25{ }^{\circ} \mathrm{C}$. The solvent gradient condition was: phase A: $0.5 \%$ formic acid in ultrapure water and phase $\mathrm{B}$ : $0.5 \%$ formic acid in acetonitrile at a flow rate of $0.5 \mathrm{~mL}$ $\mathrm{min}^{-1}$. The mass spectrometer conditions were: positive mode, $\mathrm{N}_{2}$ like dry gas, capillary $-3500 \mathrm{~V}$, scan $\mathrm{m} / \mathrm{z}$ 50-1500. The polymeric anthocyanins were identified comparing the mass spectra with data available in the literature (Blanco-Vega et al., 2014).

\section{Volatile extraction and analysis}

For the isolation and concentration of volatiles, the headspace solid-phase microextraction technique (HS-SPME) was used according to Gürbüz et al. (2006) 
with some modifications. All extractions were carried out using a DVB/CAR/PDMS fiber, of 50/30 $\mu \mathrm{m}$ film thickness. An aliquot of $10 \mathrm{~g}$ of wine was placed in $20 \mathrm{~mL}$ vials closed with Teflon cap. Vials were heated to $30{ }^{\circ} \mathrm{C}$ under agitation with a magnetic stir bar for $10 \mathrm{~min}$ for headspace equilibrium. Adsorption time was $45 \mathrm{~min}$ at the same temperature. The SPME fiber was then injected directly into a gas chromatograph mass spectrometer operating with ChemStation software. The SPME fiber was held for $10 \mathrm{~min}$ at $250{ }^{\circ} \mathrm{C}$ for desorption of volatile compounds, which were separated in HP-5MS (30 m $\times$ $0.25 \mathrm{~mm} \times 0.25 \mu \mathrm{m})$ capillary column with helium as carrier gas at constant flow of $1 \mathrm{~mL} \mathrm{~min}{ }^{-1}$. Initial oven temperature was $40{ }^{\circ} \mathrm{C}$ held for $5 \mathrm{~min}$, then increased to $160{ }^{\circ} \mathrm{C}$ at $3{ }^{\circ} \mathrm{C} \mathrm{min}{ }^{-1}$ and to $250{ }^{\circ} \mathrm{C}$ at $10{ }^{\circ} \mathrm{C} \mathrm{min}{ }^{-1}$ and kept for $10 \mathrm{~min}$ before returning to $40{ }^{\circ} \mathrm{C}$, in a total cycle of $64 \mathrm{~min}$; transfer line temperature at $250{ }^{\circ} \mathrm{C}$; MS detector in SCAN mode $30-500 \mathrm{~m} / \mathrm{z}$.

Volatile compounds were tentatively identified by comparison with the NIST library considering $70 \%$ similarity as the cut-off, further confirming the results with the retention indexes calculated according to the Kovats Index and compared to data reported on Nist Webbook (https://webbook.nist.gov), Chemspider (www. chemspider.com) or PubChem (www.pubchem.ncbi. nlm.nih.gov) websites. Only aromatic compounds with difference in Kovats Retention Indices lower than 70 units up or down were accepted. All analyses were carried out in triplicate.

\section{Statistical analysis}

The Partial Least Squares Discriminant Analysis (PLS-DA) was performed to investigate the trends or group formations of wines from different ageing times on all volatile compounds in the wine samples analyzed by the MetaboAnalyst Program (www.metaboanalyst.ca).

\section{Results}

At bottling, winter wine composition was on average $14 \%$ alcohol, $\mathrm{pH} 4.0$, fixed acidity $5.4 \mathrm{~g} \mathrm{~L}^{-1}$ tartaric acid, residual sugar $3.1 \mathrm{~g} \mathrm{~L}^{-1}$, ashes $3.9 \mathrm{~g} \mathrm{~L}^{-1}$, total anthocyanin content $380 \mathrm{mg} \mathrm{L}^{-1}$, total phenolics $2.4 \mathrm{~g} \mathrm{~L}^{-1}$, total flavanols $2.7 \mathrm{~g} \mathrm{~L}^{-1}$, color intensity of 13.5 , color hue 0.75 , and polymerized pigments $54 \%$ (Table 1 ).

Ageing decreased the total monomeric anthocyanin content and increased color hue and the polymerized pigments index, with no changes in color intensity (Tables 2 and 3). The monomeric anthocyanins identified in the Brazilian Syrah winter wine were the malvidin3-O-glucoside $(\mathrm{m} / \mathrm{z} 493)$, peonidin-3-O-glucoside $(\mathrm{m} / \mathrm{z}$ 463), petunidin-3-O-glucoside $(\mathrm{m} / \mathrm{z} 479)$ and delphinidin3-O-glucoside $(\mathrm{m} / \mathrm{z} 465)$ and the respective acylated form with coumaroyl and acetyl groups. The malvidin 3-O-glucoside was the main anthocyanin for all wine samples (Table 4), remaining 5-10\% of the concentration at bottling after 36 months of ageing.

Contribution of yellow component to the overall wine color was on average $38 \%$ at bottling reaching $41 \%$ after 42 months of ageing, while red color changed from $49 \%$ to $46 \%$ in the same period. Blue component (OD 620) was almost constant during ageing (Tables 1 and 3).

Polymerized pigments ranged from $54 \%$ at bottling to $80 \%$ after 42 months of ageing (Tables 1 and 3). Although not all wine samples were analyzed at 30 and 42 months after ageing, it seems that polymerized pigments reached maximum values after 30 months of ageing and remained constant afterwards.

Among polymerized anthocyanins, 14 pyranoanthocyanins were identified in wine samples, mainly carboxy-pyranoanthocyanins (Vitisin type), hydroxyphenyl pyranoanthocyanin and flavan-3-ol pyranoanthocyanin (Table 5). Vitisin A ( $\mathrm{m} / \mathrm{z} 561)$ formed

Table 1 - Physicochemical parameters of Syrah winter wine samples at bottling.

\begin{tabular}{|c|c|c|c|c|c|c|c|c|c|c|c|c|}
\hline \multirow{2}{*}{ Parameter } & \multicolumn{6}{|c|}{2013 winter season } & \multicolumn{3}{|c|}{2014 winter season } & \multicolumn{3}{|c|}{2015 winter season } \\
\hline & VAR13* & IND13 & SB13 & SAA13 & SSP13 & TP13 & VAR14 & TC14 & SSP14 & IT015 & TC15 & TP15 \\
\hline Alcohol (\% vol) & 13 & 13 & 14 & 14 & 15 & 13 & 14 & 15 & 16 & 14 & 15 & 14 \\
\hline $\mathrm{pH}$ & 3.83 & 3.83 & 3.99 & 3.73 & 3.94 & 3.99 & 4.08 & 3.96 & 4.27 & 4.27 & 4.03 & 4.21 \\
\hline Fixed acidity ( $\mathrm{g} \mathrm{L}^{-1}$ ) & 6.57 & 6.75 & 5.64 & 6.24 & 6.22 & 5.18 & 5.07 & 5.56 & 4.46 & 4.35 & 4.85 & 4.05 \\
\hline Residual sugar (g L-1) & 3.46 & 3.46 & 3.67 & 3.00 & 3.60 & 2.93 & 2.87 & 3.47 & 2.00 & 2.87 & 3.34 & 2.40 \\
\hline Ashes (g L-1) & 3.75 & 4.14 & 3.72 & 3.21 & 3.94 & 3.96 & 3.54 & 3.70 & 4.24 & 4.76 & 3.61 & 4.47 \\
\hline Total polyphenols ( $\mathrm{g} \mathrm{L}^{-1}$ ) & 2.41 & 2.51 & 3.30 & 2.86 & 2.80 & 2.49 & 2.28 & 1.78 & 2.50 & 2.30 & 1.74 & 1.95 \\
\hline Total anthocyanins (mg L-1) & 540.11 & 427.18 & 414.61 & 399.20 & 443.29 & 363.80 & 358.47 & 200.98 & 306.50 & 451.22 & 293.31 & 360.70 \\
\hline Total flavanols (g L-1) & 2.71 & 2.87 & 2.98 & 2.97 & 3.63 & 3.53 & 2.34 & 1.98 & 2.88 & 2.56 & 1.95 & 2.42 \\
\hline Color intensity & 14.32 & 15.48 & 15.49 & 15.72 & 17.59 & 12.73 & 12.42 & 10.63 & 14.98 & 10.90 & 10.18 & 11.82 \\
\hline Color hue & 0.61 & 0.64 & 0.71 & 0.65 & 0.70 & 0.73 & 0.71 & 0.78 & 0.86 & 0.86 & 0.82 & 0.88 \\
\hline OD $420 \%$ & 33 & 34 & 36 & 34 & 36 & 36 & 36 & 39 & 40 & 39 & 39 & 40 \\
\hline OD $520 \%$ & 54 & 53 & 51 & 53 & 51 & 50 & 50 & 49 & 46 & 46 & 48 & 46 \\
\hline OD $620 \%$ & 12 & 12 & 13 & 13 & 13 & 13 & 14 & 12 & 14 & 15 & 13 & 14 \\
\hline Polymerized pigments (\%) & 47 & 52 & 52 & 51 & 54 & 53 & 45 & 64 & 61 & 52 & 59 & 60 \\
\hline
\end{tabular}

Average values of three samples per region. *Number represents the harvest season; OD = optical density at 420, 520, or $620 \mathrm{~nm}$; VAR = Vargem (SP); IND = Indaiatuba (SP); SB = São Bento do Sapucaí (SP); SAA = Santo Antônio do Amparo (MG); SSP = São Sebastião do Paraíso (MG); TP = Três Pontas (MG); TC = Três Corações (MG); ITO = Itobi (SP). 
Table 2 - Physicochemical parameters of Syrah winter wine samples after 20 months of ageing in bottle.

\begin{tabular}{|c|c|c|c|c|c|c|c|c|c|c|c|c|}
\hline \multirow{2}{*}{ Parameter } & \multicolumn{6}{|c|}{2013 winter season } & \multicolumn{3}{|c|}{2014 winter season } & \multicolumn{3}{|c|}{2015 winter season } \\
\hline & VAR13* & IND13 & SB13 & SAA13 & SSP13 & TP13 & VAR14 & TC14 & SSP14 & IT015 & TC15 & TP15 \\
\hline Alcohol (\% vol) & 14 & 14 & 14 & 14 & 15 & 13 & 14 & 15 & 16 & 14 & 15 & 14 \\
\hline $\mathrm{pH}$ & 3.78 & 3.79 & 3.91 & 3.75 & 3.93 & 3.85 & 4.09 & 3.90 & 4.16 & 4.14 & 3.99 & 4.13 \\
\hline Fixed acidity $\left(\mathrm{g} \mathrm{L}^{-1}\right)$ & 5.75 & 6.16 & 5.60 & 5.90 & 5.43 & 5.33 & 5.27 & 5.99 & 5.12 & 4.80 & 5.47 & 5.00 \\
\hline Residual sugar (g L $\mathrm{L}^{-1}$ ) & 3.40 & 2.53 & 2.14 & 3.27 & 3.33 & 2.93 & 2.20 & 3.20 & 2.94 & 2.80 & 3.13 & 2.87 \\
\hline Ashes (g $\left.\mathrm{L}^{-1}\right)$ & 4.09 & 4.26 & 3.80 & 3.34 & 4.10 & 4.15 & 3.56 & 3.63 & 4.41 & 4.71 & 3.64 & 4.43 \\
\hline Total polyphenols ( $\mathrm{g} \mathrm{L}^{-1}$ ) & 2.06 & 2.36 & 2.40 & 2.51 & 2.87 & 1.90 & 2.09 & 1.82 & 2.54 & 2.29 & 1.89 & 2.13 \\
\hline Total anthocyanins (mg L-1) & 167.83 & 147.76 & 136.82 & 155.87 & 156.50 & 116.27 & 112.85 & 57.21 & 122.97 & 187.25 & 100.55 & 135.74 \\
\hline Total flavanols $\left(\mathrm{g} \mathrm{L}^{-1}\right)$ & 2.68 & 3.11 & 2.90 & 3.02 & 3.65 & 2.85 & 2.50 & 1.98 & 3.30 & 2.61 & 2.10 & 2.52 \\
\hline Color intensity & 14.33 & 14.67 & 14.90 & 15.12 & 17.22 & 12.43 & 12.32 & 12.63 & 14.98 & 12.73 & 11.73 & 12.86 \\
\hline Color hue & 0.72 & 0.73 & 0.76 & 0.75 & 0.77 & 0.78 & 0.88 & 0.84 & 0.89 & 0.89 & 0.84 & 0.89 \\
\hline OD $420 \%$ & 37 & 37 & 38 & 37 & 38 & 38 & 40 & 40 & 41 & 40 & 40 & 41 \\
\hline OD $520 \%$ & 50 & 50 & 49 & 50 & 49 & 49 & 45 & 48 & 46 & 46 & 47 & 46 \\
\hline OD $620 \%$ & 13 & 13 & 13 & 13 & 14 & 13 & 15 & 12 & 13 & 14 & 13 & 13 \\
\hline Polymerized pigments (\%) & 69 & 69 & 72 & 68 & 73 & 72 & 73 & 83 & 78 & 72 & 78 & 77 \\
\hline
\end{tabular}

Average values of three samples per region. *Number represents the harvest season; OD = optical density at 420, 520, or $620 \mathrm{~nm}$; VAR = Vargem (SP); IND = Indaiatuba (SP); SB = São Bento do Sapucaí (SP), SAA = Santo Antônio do Amparo (MG); SSP = São Sebastião do Paraíso (MG), TP = Três Pontas (MG), TC = Três Corações (MG); ITO = Itobi (SP).

Table 3 - Physicochemical parameters of Syrah winter wine samples after 30 and 42 months of ageing in bottle.

\begin{tabular}{|c|c|c|c|c|c|c|c|}
\hline \multirow{2}{*}{ Parameter } & \multicolumn{2}{|c|}{30 months ageing } & \multicolumn{5}{|c|}{42 months ageing } \\
\hline & SSP14* & TC14 & IND13 & SB13 & SAA13 & SSP13 & TP13 \\
\hline Alcohol (\% vol) & 16 & 15 & 14 & 14 & 14 & 15 & 13 \\
\hline $\mathrm{pH}$ & 4.11 & 3.84 & 3.79 & 3.89 & 3.72 & 3.92 & 3.83 \\
\hline Fixed acidity ( $\left.\mathrm{g} \mathrm{L}^{-1}\right)$ & 4.99 & 6.03 & 5.86 & 5.37 & 5.58 & 5.57 & 5.84 \\
\hline Residual sugar (g L-1) & 2.73 & 2.80 & 2.93 & 1.87 & 2.73 & 4.00 & 2.53 \\
\hline Ashes $\left(\mathrm{g} \mathrm{L}^{-1}\right)$ & 4.25 & 3.57 & 4.07 & 3.62 & 3.05 & 3.85 & 3.83 \\
\hline Total polyphenols ( $\mathrm{g} \mathrm{L}^{-1}$ ) & 2.57 & 1.77 & 2.41 & 2.54 & 2.60 & 3.00 & 2.18 \\
\hline Total anthocyanins (mg L-1) & 90.06 & 42.32 & 74.94 & 67.26 & 82.71 & 82.25 & 55.17 \\
\hline Total flavanols (g $\mathrm{L}^{-1}$ ) & 2.96 & 1.88 & 3.08 & 3.36 & 3.43 & 3.74 & 2.64 \\
\hline Color intensity & 15.65 & 12.42 & 14.61 & 14.79 & 14.72 & 17.45 & 12.91 \\
\hline Color hue & 0.93 & 0.86 & 0.86 & 0.88 & 0.87 & 0.89 & 0.89 \\
\hline OD $420 \%$ & 42 & 41 & 40 & 41 & 40 & 41 & 41 \\
\hline OD $520 \%$ & 45 & 47 & 46 & 46 & 46 & 46 & 46 \\
\hline OD $620 \%$ & 13 & 12 & 13 & 13 & 13 & 13 & 12 \\
\hline Polymerized pigments (\%) & 80 & 85 & 80 & 83 & 79 & 81 & 83 \\
\hline
\end{tabular}

Average values of three samples per region. *Number represents the harvest season. OD = optical density at 420, 520, or $620 \mathrm{~nm}$; IND = Indaiatuba (SP); SB = São Bento do Sapucaí (SP); SAA = Santo Antônio do Amparo (MG); SSP = São Sebastião do Paraíso (MG); TP = Três Pontas (MG); TC = Três Corações (MG).

Table 4 - Mass spectra and concentration of monomeric anthocyanins of Syrah winter wine of five regions at bottling and after 19 and 36 months of ageing in bottle.

\begin{tabular}{|c|c|c|c|c|c|c|c|c|c|c|}
\hline & & Peak & RT & {$[\mathrm{M}]^{+}(\mathrm{m} / \mathrm{z})$} & MS/MS (m/z) & SB13 & TP13 & IND13 & SAA13 & SSP13 \\
\hline & & & $\min$ & & & & & & & \\
\hline \multirow{6}{*}{$\mathrm{T}_{0}$} & Delphinidin-3-glu & 1 & 12.3 & 465 & 303 & $17.9 \pm 1.2$ & nd & $10.4 \pm 0.1$ & $15.4 \pm 0.6$ & $19.2 \pm 0.8$ \\
\hline & Petunidin-3-glu & 3 & 14.3 & 479 & 317 & $34.3 \pm 2.12$ & $24.6 \pm 1.7$ & $23.4 \pm 0.2$ & $26.0 \pm 0.3$ & $35.0 \pm 0.6$ \\
\hline & Malvidin-3-glu & 5 & 15.6 & 493 & 331 & $269.0 \pm 19.3$ & $266.0 \pm 22.9$ & $163.7 \pm 1.7$ & $114.5 \pm 2.1$ & $168.2 \pm 0.8$ \\
\hline & Petunidin-3-acgly & 8 & 21.8 & 521 & 317 & $10.3 \pm 0.5$ & nd & $6.6 \pm 0.1$ & nd & $7.2 \pm 0.5$ \\
\hline & Malvidin-3-acgly & 10 & 22.2 & 535 & 331 & nd & nd & $22.0 \pm 1.2$ & $15.3 \pm 0.9$ & nd \\
\hline & Peonidin-3-cmgly & 14 & 25.6 & 609 & 301 & $22.4 \pm 1.0$ & $20.3 \pm 1.6$ & $17.5 \pm 0.3$ & $16.0 \pm 0.1$ & $9.1 \pm 1.1$ \\
\hline \multirow{2}{*}{$\mathrm{T}_{19}$} & Malvidin-3-glu & 5 & 15.6 & 493 & 331 & $42.1 \pm 2.3$ & $37.8 \pm 3.8$ & $31.0 \pm 0.9$ & $25.7 \pm 2.7$ & $28.6 \pm 1.3$ \\
\hline & Petunidin-3-acgly & 8 & 21.8 & 521 & 317 & $7.5 \pm 0.7$ & $3.2 \pm 0.01$ & $7.5 \pm 0.4$ & nd & $9.6 \pm 0.3$ \\
\hline \multirow{2}{*}{$\mathrm{T}_{36}$} & Malvidin-3-glu & 5 & 15.6 & 493 & 331 & $16.3 \pm 1.1$ & $15.3 \pm 0.4$ & nd & $11.7 \pm 1.1$ & $17.6 \pm 1.8$ \\
\hline & Petunidin-3-acgly & 8 & 21.8 & 521 & 317 & $3.7 \pm 0.2$ & $2.9 \pm 0.0$ & $4.5 \pm 0.2$ & nd & $5.1 \pm 0.5$ \\
\hline
\end{tabular}

$\mathrm{RT}=$ retention time; glu = glucoside; acgly = 6"-acetyl-glycoside; cmgly = (6-p-coumaroyl) glycoside; nd = not detected; $\mathrm{T}_{0}=$ wine at bottling; $\mathrm{T}_{19}=$ wine after 19 months of ageing; $T_{36}=$ wine after 36 months of ageing. Petunidin-3-acgly, malvidin-3-acgly and peonidin-3-cmgl were expressed as petunidin-3-glucoside, malvidin-3glucoside and peonidin-3-glucoside equivalent, respectively. Results expressed as $\mathrm{mg} 100 \mathrm{~mL}^{-1}$; IND = Indaiatuba; SB = São Bento do Sapucaí; SAA = Santo Antônio do Amparo; SSP = São Sebastião do Paraíso; TP = Três Pontas. 
from malvidin 3-O-glucoside and pyruvic acid reaction was the most abundant pyranoanthocyanins (He et al., 2012). Ageing decreased the content of vitisin A on average by $10-30 \%$ in most samples, while 10-HP-pymv3-gly ( $\mathrm{m} / \mathrm{z} 609)$ increased during ageing (Table 6).

The Partial Least Squares Discriminant Analysis (PLS-DA) accounted for $63 \%$ of the total data variance. The two-dimensional graph showed a clear separation of aromatic compounds from bottled wines to those over 30 months ageing (Figure 1).

Table 7 summarizes all the aromatic volatile compounds tentatively identified in the samples, regardless of vineyard and ageing. Esters represented the principal class of compounds with 40 aromatic volatile compounds identified, followed by terpenes (17 compounds), benzene (14), and alcohol (12). Esters were found mainly at bottling, but their concentration increased slightly at 30 months ageing.

\section{Discussion}

Winter wines composition resemble that of Syrah wines from traditional regions, such as Australia (Antalick et al., 2015), Italy (Condurso et al., 2016), California (Brillante et al., 2018), and South Africa (Hunter and Volschenk, 2018) confirming the great potential of this technique for Brazilian viticulture.

The anthocyanins identified in the Brazilian Syrah winter wines were 3-O-glucoside and acylated forms of malvidin, peonidin, petunidin, and delphinidin also described in Syrah wines from Spain (Blanco-Vega et al., 2014).

Table 5 - Mass spectra of pyranoanthocyanins of Syrah winter wine of five regions at bottling and after 19 and 36 months of ageing in bottle.

\begin{tabular}{|c|c|c|c|c|}
\hline & Compound & RT & $\mathrm{MS}(\mathrm{m} / \mathrm{z})$ & MS2 (m/z) \\
\hline \multirow{8}{*}{$\begin{array}{l}\text { A-type vitisins (10-carboxy- } \\
\text { pyranoanthocyanins) }\end{array}$} & & $\min$ & & \\
\hline & 10-Carboxy-pymv-3-glc (vitisin A) & 18.3 & 561 & 399 \\
\hline & 10-Carboxy-pymv-3-acglc (ac-vitisin A) & 19.9 & 603 & 399 \\
\hline & 10-Carboxy-pymv-3-cmglc (cm-vitisin A) & 23.6 & 707 & 399 \\
\hline & 10-Carboxy-pypn-3-glc & 16.9 & 531 & 369 \\
\hline & 10-Carboxy-pypn-3-cmglc & 22.9 & 677 & 369 \\
\hline & 10-Carboxy-pypt-3-glc & 14.4 & 547 & 385 \\
\hline & 10-Carboxy-pydp-3-glc & 10.4 & 533 & 371 \\
\hline B-type vitisins (pyranoanthocyanins) & pymv-3-glc (vitisin B) & 19.4 & 517 & 355 \\
\hline \multirow{4}{*}{ 10-Hydroxyphenyl-pyranoanthocyanins } & 10-HP-pymv-3-glc & 34 & 609 & 447 \\
\hline & 10-HP-pymv-3-acglc & 36.5 & 651 & 447 \\
\hline & 10-HP-pymv-3-cmglc & 38.6 & 755 & 447 \\
\hline & 10-DHP-pymv-3-glc & 31.3 & 625 & 463 \\
\hline \multirow{2}{*}{ 10-Flavanol-pyranoanthocyanins } & 10-(+)-Catechin-pypn-3-acglc & 28.4 & 817 & $665,613,461$ \\
\hline & 10-(-)-Epicatechin-pypn-3-acglc & 30.5 & 817 & $665,613,461$ \\
\hline
\end{tabular}

Table 6 - Relative percentage of polymeric anthocyanins in winter wine of five regions at bottling and after 19 and 36 months of ageing in bottle.

\begin{tabular}{|c|c|c|c|c|c|c|c|c|c|c|c|c|c|c|c|}
\hline & \multicolumn{3}{|c|}{ SB13 } & \multicolumn{3}{|c|}{ TP13 } & \multicolumn{3}{|c|}{ IND13 } & \multicolumn{3}{|c|}{ SAA13 } & \multicolumn{3}{|c|}{ SSP13 } \\
\hline & $\mathrm{T}_{0}$ & $\mathrm{~T}_{19}$ & $T_{36}$ & $T_{0}$ & $\mathrm{~T}_{19}$ & $\mathrm{~T}_{36}$ & $\mathrm{~T}_{0}$ & $\mathrm{~T}_{19}$ & $\mathrm{~T}_{36}$ & $\mathrm{~T}_{0}$ & $\mathrm{~T}_{19}$ & $\mathrm{~T}_{36}$ & $\mathrm{~T}_{0}$ & $\mathrm{~T}_{19}$ & $\mathrm{~T}_{36}$ \\
\hline 10-Carboxy-pypt-3-gly & 3 & 4 & 3 & 1 & 2 & nd & 2 & 5 & 5 & 3 & 4 & 4 & 3 & 7 & 7 \\
\hline 10-Carboxy-pypn-3-gly & 6 & 6 & 5 & 4 & 4 & 4 & 5 & 7 & 7 & 6 & 6 & 6 & 7 & 8 & 7 \\
\hline 10-Carboxy-pymv-3-gly (vitisin A) & 45 & 41 & 32 & 25 & 20 & 17 & 45 & 54 & 50 & 32 & 32 & 26 & 44 & 45 & 41 \\
\hline 10-Carboxy-pymv-3-acgly (ac-vitisin A) & 5 & 4 & 3 & 6 & 4 & 3 & 6 & 14 & 14 & 9 & 7 & 5 & 12 & 11 & 9 \\
\hline 10-Carboxy-pypn-3-cmgly & 2 & 2 & 2 & 2 & 2 & nd & 15 & 3 & 3 & 3 & 3 & 3 & 3 & 3 & 3 \\
\hline 10-Carboxy-pymv-3-cmgly (cm-vitisin A) & 7 & 8 & 8 & 6 & 5 & 5 & 3 & 8 & 9 & 7 & 7 & 8 & 8 & 8 & 10 \\
\hline TOTAL Carboxy- & 68 & 65 & 53 & 44 & 37 & 29 & 76 & 91 & 88 & 60 & 59 & 52 & 77 & 82 & 77 \\
\hline 10-(+)-Catechin-pypn-3-acgly & 2 & 2 & 5 & 4 & 3 & 4 & 4 & 3 & 2 & 5 & 3 & 3 & 3 & 3 & 3 \\
\hline 10-(-)-Epicatechin-pypn-3-acgly & 6 & 5 & 6 & nd & nd & nd & nd & nd & nd & 8 & 10 & 9 & nd & nd & nd \\
\hline TOTAL Flavanol- & 8 & 7 & 11 & 4 & 3 & 4 & 4 & 3 & 2 & 13 & 13 & 12 & 3 & 3 & 3 \\
\hline 10-HP-pymv-3-gly & 17 & 17 & 25 & 31 & 34 & 36 & 8 & 9 & 7 & 17 & 17 & 22 & 13 & 10 & 13 \\
\hline 10-HP-pymv-3-acgly & 3 & 2 & 3 & 6 & 5 & 5 & 3 & 2 & 2 & 5 & 4 & 4 & 4 & 2 & 3 \\
\hline 10-HP-pymv-3-cmgly & 3 & 3 & 5 & 8 & 7 & 6 & 2 & nd & 2 & 5 & 4 & 5 & 4 & 3 & 3 \\
\hline 10-DHP-pymv-3-gly & - & 4 & 4 & 8 & 16 & 19 & nd & nd & nd & nd & 3 & 4 & nd & 1 & 3 \\
\hline TOTAL Hydroxyphenyl- & 23 & 26 & 37 & 53 & 62 & 66 & 13 & 11 & 11 & 27 & 28 & 35 & 21 & 16 & 22 \\
\hline
\end{tabular}

$\mathrm{T}_{0}=$ wine at bottling; $\mathrm{T}_{19}=$ wine after 19 months of ageing; $\mathrm{T}_{36}=$ wine after 36 months of ageing. Gly = glycoside; acgly = 6"-acetyl-glycoside; cmgly = (6-p-coumaroyl) glycoside; pymv = pyranomalvidin; pypn = pyranopeonidin; pypt = pyranopetunidin; pydp = pyranodelphinidin; IND = Indaiatuba; SB = São Bento do Sapucaí; SAA = Santo Antônio do Amparo; SSP = São Sebastião do Paraíso; TP = Três Pontas. 


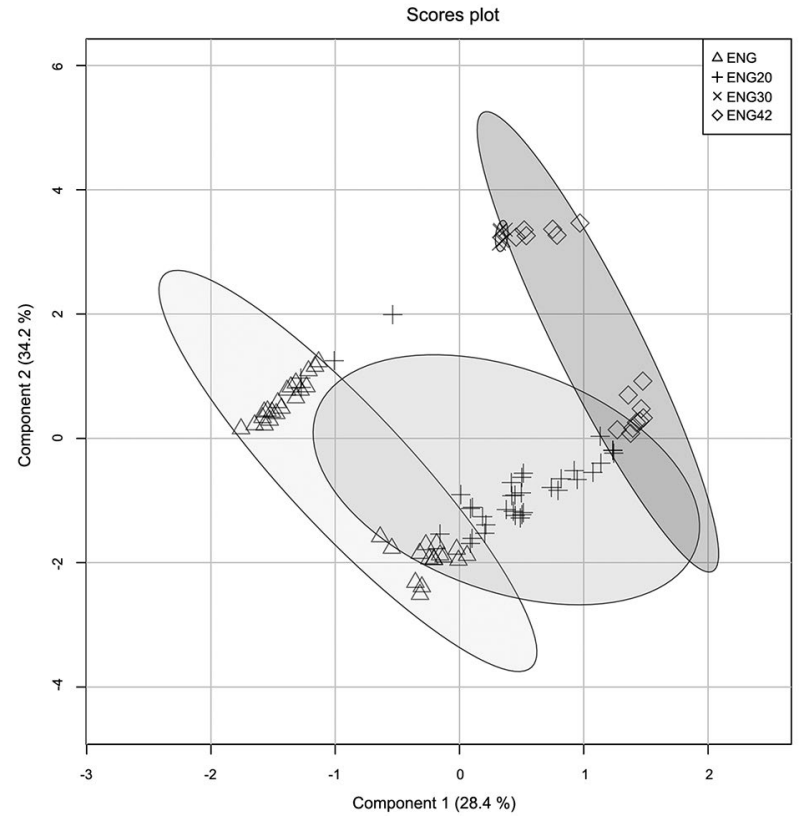

Figure 1 - Discriminant Analysis of aromatic volatile compounds of Syrah wines at bottling (ENG) and after ageing for 20 months (ENG20), 30 months (ENG30), or 42 months (ENG42).
It is well known that a fraction of the anthocyanin pigments disappears rapidly few months after fermentation. These pigments may be either broken down by external factors (temperature, light, oxygen), precipitated in colloidal coloring matter, combined and condensed with tannins, and also forming stable anthocyanin derived pigments, named pyranoanthocyanins. These pigments are produced by reaction between anthocyanins and acetaldehyde, pyruvic acid or vinylphenols, by-products of yeast, in young wine and also from condensation between anthocyanin and/or flavan-3-ols in aged wine (He et al., 2012). The elimination of anthocyanins leads to color loss and is detrimental to wine while pyranoanthocyanins production form more stable molecules responsible for color maintenance (Cheynier et al., 2006).

A reduction in total anthocyanins from 490 to $60 \mathrm{mg} \mathrm{L}^{-1}$ followed by an intense reduction in color intensity from bottling to 17 months aged was observed in Italian Syrah wines (Condurso et al., 2016). Color intensity in Brazilian Syrah winter wines, on the other hand, was not affected during ageing as well as total phenolic compounds and flavanols, which remained almost constant through ageing. Moreover, there was an increase in hydroxyphenyl pyranoanthocyanins and

Table 7 - Aromatic volatile compounds tentatively identified in the Syrah winter wine samples.

\begin{tabular}{|c|c|c|c|c|}
\hline Compound & Code & CAS & Kovatsa & Odor ${ }^{b}$ \\
\hline \multicolumn{5}{|l|}{ Acids } \\
\hline Hexanoic acid & $\mathrm{BF}$ & $142-62-1$ & 997 & Rancid, sour, sharp, pungent, cheesy, fatty \\
\hline n-decanoic acid & BJ & $334-48-5$ & 1374 & Unpleasant rancid, sour, fatty, citrus, soapy \\
\hline Octanoic acid & $\mathrm{BC}$ & $124-07-2$ & 1194 & Fruity-acid \\
\hline \multicolumn{5}{|l|}{ Alkanes } \\
\hline hexadecane & BW & $544-76-3$ & 1599 & Mild wax \\
\hline tetradecane & $\mathrm{CH}$ & $629-59-4$ & 14001 & Mild wax \\
\hline \multicolumn{5}{|l|}{ Alcohols } \\
\hline 1-decanol & $A Q$ & $112-30-1$ & 1275 & Sweet, fat-like \\
\hline 1-heptanol & AM & $111-70-6$ & 977 & Woody, oily, fatty \\
\hline 1-hexanol & $\mathrm{AL}$ & $111-27-3$ & 877 & Herbaceous, woody, sweet, green fruit, banana, flower, grass \\
\hline 1-hexanol, 2-ethyl & $x$ & $104-76-7$ & 1035 & Oily, sweet, flowery, rose, green \\
\hline 1-nonanol & $\mathrm{BH}$ & $143-08-8$ & 1178 & Rose-orange, fat, floral, green, oil \\
\hline 1-octanol & $\mathrm{AO}$ & $111-87-5$ & 1076 & Fresh, orange-rose, sweet, bitter almond, burnt matches, fat, floral \\
\hline 1-octen-3-ol & СT & $3391-86-4$ & 984 & Sweet, earthy, herbaceous lavender, rose, cucumber, fat, floral, mushroom \\
\hline 2-heptanol & BV & $543-49-7$ & 905 & Brassy, herbaceous, fruity, green, citrus, earth, mushroom, oil \\
\hline 2-nonanol & CG & $628-99-9$ & 1102 & Waxy, green, creamy, citrus, orange, cheese, fruity \\
\hline Cis-3-hexen-1-ol & CK & $928-96-1$ & 865 & Grassy-green, herbaceous, leafy \\
\hline 3-nonen-1-ol, (Z) & DB & $10340-23-5$ & 1158 & Fresh, waxy, green, melon, rind, tropical, mushroom \\
\hline 3-octanol & BY & $589-98-0$ & 998 & Sweet, oily, nutty, herbaceous \\
\hline \multicolumn{5}{|l|}{ Aldehydes } \\
\hline Decanal & AR & $112-31-2$ & 1209 & Sweet, waxy, flowery, citrus, fatty \\
\hline Furfural & $\mathrm{N}$ & $98-01-1$ & 841 & Sweet, woody, almond, fragrant, baked, bread \\
\hline Nonanal & $\mathrm{BD}$ & $124-19-6$ & 1105 & Fatty, citrus-like \\
\hline benzeneacetaldehyde & AV & $122-78-1$ & $1047 \vdash$ & Harsh, green \\
\hline \multicolumn{5}{|l|}{ Benzenes } \\
\hline benzaldehyde & $\mathrm{T}$ & $100-52-7$ & 964 & Bitter almond \\
\hline
\end{tabular}


Table 7 - Continuation.

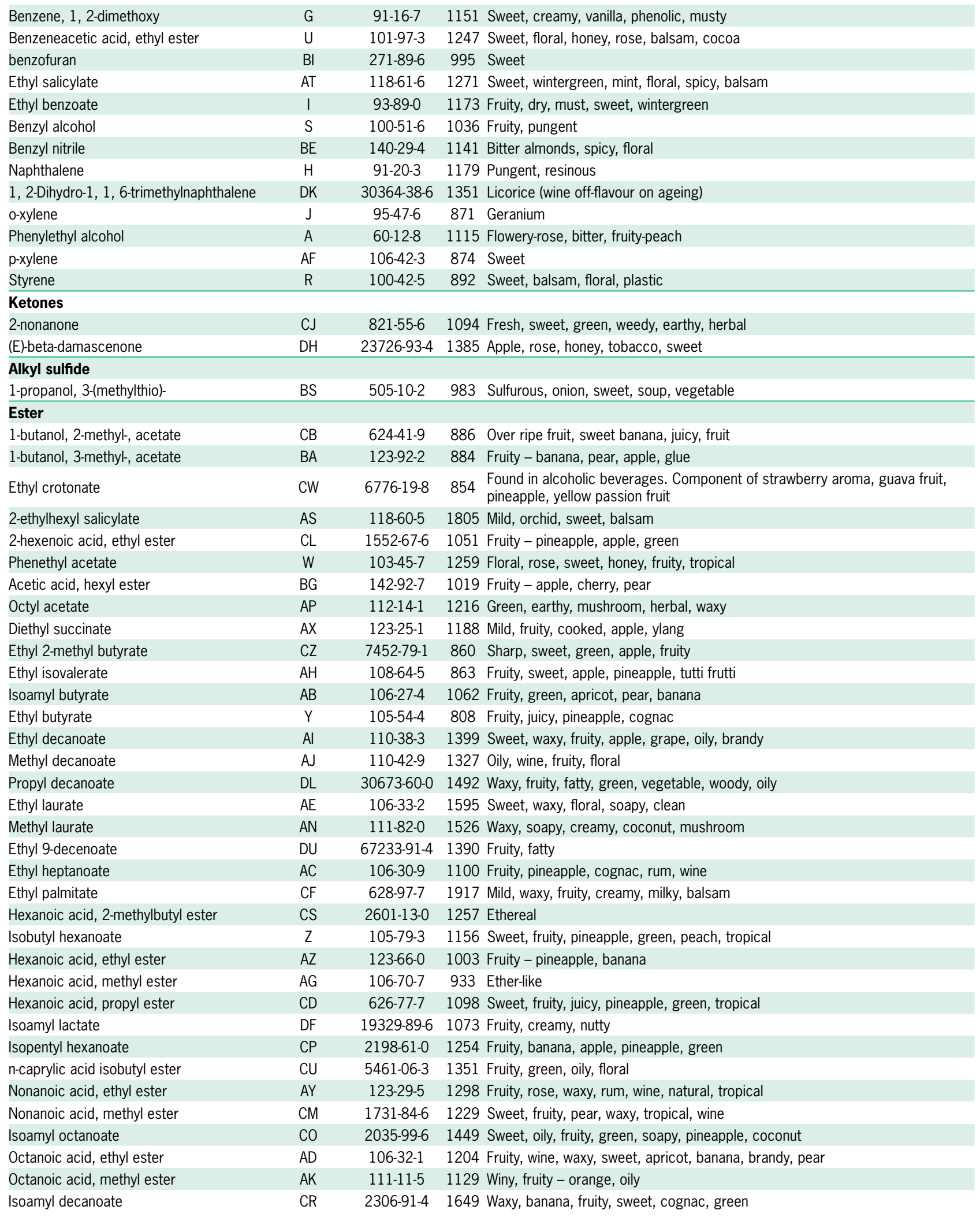


Table 7 - Continuation.

\begin{tabular}{|c|c|c|c|}
\hline Pentadecanoic acid, ethyl ester & DN & $41114-00-5 \quad 1871$ & Honey, sweet \\
\hline Ethyl lactate & M & $97-64-3$ & Sharp, tart, fruity, buttery, butterscotch \\
\hline Propyl octanoate & $\mathrm{CA}$ & 624-13-5 1294 & Coconut, caco, gin \\
\hline Tetradecanoic acid, ethyl ester & $\mathrm{BB}$ & $124-06-1$ & Sweet, waxy, violet, orris \\
\hline Undecanoic acid, ethyl ester & CE & $627-90-7$ & Soapy, waxy, fatty, cognac, coconut \\
\hline \multicolumn{4}{|l|}{ ether } \\
\hline Geranyl ethyl ether & DM & $40267-72-9 \quad 1290$ & Diffusive, ethereal, fruity, green \\
\hline \multicolumn{4}{|l|}{ Volatile phenols } \\
\hline Phenol, 2, 4-bis (1, 1-dimethylethyl) & $\mathrm{L}$ & $96-76-4$ & phenolic \\
\hline Phenol, 2-methoxy- & $\mathrm{F}$ & $90-05-1$ & Phenolic, smoke, spice, vanilla woody \\
\hline Phenol, 4-ethyl- & AW & $123-07-9$ & Phenolic, castoreum, smoke, guaiacol \\
\hline \multicolumn{4}{|l|}{ Furans } \\
\hline Ethyl 2-furoate & $\mathrm{BZ}$ & $614-99-3$ & Ethyl benzoate, fruity, floral \\
\hline \multicolumn{4}{|l|}{ Lactones } \\
\hline butyrolactone & K & $96-48-0$ & Sweet, aromatic, buttery, creamy, oily, fatty, caramel \\
\hline \multicolumn{4}{|l|}{ Monoterpenes } \\
\hline .alpha.-terpineol & 0 & $98-55-5$ & Pine-like, woody, resinous \\
\hline Laevo-camphor & $\mathrm{BL}$ & $\begin{array}{cc}\text { 464-48-2 } & 1145- \\
\mathrm{NF}\end{array}$ & Camphor \\
\hline citronellol & AA & $106-22-9 \quad 1232$ & Flowery - rose \\
\hline menthol & DE & $15356-70-4 \quad 1176$ & Mint-like \\
\hline$( \pm)$ menthone & $\mathrm{D}$ & $89-80-5 \quad 1155$ & Mint-like \\
\hline D-Limonene & $\mathrm{CV}$ & $5989-27-5 \quad 1030$ & Citrus, orange, fresh, sweet \\
\hline Levomenthol & CQ & 2216-51-5 1174 & Peppermint, cooling, mentholic, minty \\
\hline I-menthone & $\mathrm{DD}$ & $14073-97-3 \quad 1155$ & Deep menthol, peppermint, herbal, camphor \\
\hline Methyl salicylate & $\mathrm{AU}$ & $119-36-8 \quad 1193$ & Wintergreen, mint \\
\hline p-Cymene & $\mathrm{P}$ & $99-87-6$ & Fresh, citrus, terpene, woody, spice \\
\hline Terpinen-4-ol & $\mathrm{BX}$ & $562-74-3$ & Pepper, woody, earth, musty, sweet \\
\hline Linalool & B & 78-70-6 & Citrus, floral, sweet, bois de rose, woody, green, blueberry \\
\hline Eucalyptol & BM & $470-82-6 \quad 1033$ & Camphoraceous \\
\hline Beta-cyclocitral & BK & $432-25-7$ & Tropical, saffron, herbal, clean, rose, oxide, sweet, tobacco, damascene, fruity \\
\hline \multicolumn{4}{|l|}{ Sesquiterpenes } \\
\hline Calamenene & BO & 483-77-2 1522 & Herb, spice \\
\hline .alpha.-Calacorene & DG & 21391-99-1 1543 & woody \\
\hline .alpha.-Muurolene & $\mathrm{DA}$ & 10208-80-7 1499 & woody \\
\hline \multicolumn{4}{|l|}{ Pirane } \\
\hline Nerol oxide & $\mathrm{CN}$ & $1786-08-9 \quad 1158$ & Green, weedy, cortex, herbal, diphenyl oxide, narcissus, celery \\
\hline \multicolumn{4}{|l|}{ Tetrahydrothiophene } \\
\hline Blackberry thiophenone & DC & $13679-85-1998$ & Sulfur, fruity, berry \\
\hline
\end{tabular}

flavan-3-ol pyranoanthocyanins in aged wine, pigments probably responsible for the red color of wine.

The polymerized pigment index, applied to define the percentage of free and combined anthocyanins producing color in wine (Harbertson and Spayd, 2006), increased from $54 \%$ at bottling to $80 \%$ after 42 months of ageing, corroborating the contribution of copigmentation reactions to preserve color of winter wines.

The literature does not report aroma compounds in Syrah winter wines. According to Condurso et al. (2016), tipicity and quality of the wine are closely related to volatile aroma compounds from grape and those formed during the vinification process. Syrah wine has been described with spicy, dark fruit, or berry like flavors depending on the terroir. Therefore, studies report different volatile data. For example, rotundone, the sesquiterpene compound responsible for the peppery character of Syrah wines, requires an optimized procedure of extraction and therefore is not always found in Syrah wines samples (Siebert et al., 2008; Cincotta et al., 2015; Condurso et al., 2016).

Freshly fermented wines from vineyards in southeastern Brazil were combined in the left part of the PCA plot while aged wines were displaced to the right part, with positive scores (Figure 1). Loscos et 
al. (2010) also observed such tendency. The second component reflects vineyard site importance, which will be discussed in another study.

In an attempt to differentiate wine varieties by their volatile compounds profile, Fabani et al. (2013) selected four ester compounds ethyl caprylate (10632-1), diethyl succinate (123-25-1), ethyl caproate (123-66-0), and isopentyl acetate (123-92-2), one benzene compound, benzyl alcohol (100-51-6) and one alcohol, 1-hexanol (111-27-3) as representative volatile compounds in wines. All these compounds were also found in Syrah winter wines. As mentioned by these authors, ethyl caprylate was also found through wine ageing, but mainly at bottling and in winter wines aged 20 months.

Volatile esters play a significant role in wine aroma, as they are associated to "fruity" and "floral" flavors. Numerous esters compounds were identified in wine samples from thinned and control plants of Syrah vineyards located in Palermo, Italy (Condurso et al., 2016). The most represented esters identified by these authors, ethyl hexanoate (CAS 123-66-0), ethyl octanoate (CAS 106-32-1), and ethyl decanoate (CAS 110-38-3) were also present in Syrah winter wines from southeastern Brazil. However, those with the most contrasting patterns among ageing were 1-butanol, 2-methyl-acetate (624-41-9), 2-hexenoic acid, ethyl ester (1552-67-6), acetic acid, hexyl ester (142-92-7), ethyl heptanoate (106-30-9), hexanoic acid, propyl ester (626-77-7), n-caprylic acid isobutyl ester (5461-063), isoamyl decanoate (2306-91-4) identified as fruity aroma compounds, and hexanoic acid, methylbutyl ester (2601-13-0) and hexanoic acid, methyl ester (10670-7) with ether-like notes.

Diethyl succinate (123-25-1), an ester mentioned as a chemical marker of wine ageing (Fabani et al., 2013) was found after 30 and 42 months ageing, mainly in winter wines aged for 30 months. Isopentyl acetate (123-92-2) another ester, responsible for the banana bouquet, was present at bottling and after 30 months of ageing. Fabani et al. (2013) reported a tendency to find lower levels with ageing in Syrah wines. As our results are qualitatively, we were not able to measure its content in the samples; however, it was found from bottling throughout ageing, with lower amounts after 42 months in the bottle.

Furfural (98-01-1), an aldehyde responsible for almond and caramel aroma, was found in Syrah winter wines over 30 months of age. This volatile compound is formed from carbohydrates during wine ageing; however, it can also be generated from hemicelluloses of the barrels (Condurso et al., 2016).

Among benzene class, benzyl alcohol (10051-6) and ethyl benzoate (93-89-0) associated to 'flowery' and 'sweet' aromas were also found mainly at 30 months of ageing as well as 1,2-Dihydro-1, 1, 6-trimethylnaphthalene (30364-38-6), a benzene compound known as off-flavor of ageing. Benzene levels decreased from bottle to 20 months ageing and then increased at 30 months with great loss at 42 months.

The presence of leafy and herbaceous aromas from C6 compounds such as cis-3-hexen-1-ol (928-96-1) released from the enzymatic degradation of lipids from grape cell membrane (Brillante et al., 2018) is related to fresh grape processing. Indeed this compound was found mainly at bottling in Syrah winter wines. Volatile compounds belonging to alcohol, alkyl sulfide, and acids classes were found mainly in young wines.

The volatile phenols associated with smoke, spice, and phenolic aromas (Loscos et al., 2010), guaiacol (90-05-1), and 4-ethylphenol (123-07-9) increased their concentration during ageing.

Terpenes are synthesized during grape maturation. They have pleasant flavor perceived even at low concentrations due to its very low olfactory threshold. The fermentation process has little contribution on terpene levels and therefore their content depends on vineyard management /Condurso et al., 2016). Syrah winter wines showed an increase in monoterpenes until 30 months ageing with a sharp decrease at 42 months, as observed by Loscos at al. (2010) under accelerated ageing process. Citronellol (106-22-9), the rose-like aroma, was found at bottling while D-limonene (5989-27-5) and p-cymene (99-87-6), both contributing to fresh, citrus-like aroma, increased at 30 months ageing. Pepper and peppermint aromas of terpinen-4-ol (562-74-3) and levomenthol (5989-275) were found mainly at bottling and at 30 months of ageing, while l-menthone (14073-97-3) content was higher in wines for 30 months aged.

The monoterpene linalool (78-70-6) and the ketone $\beta$-damascenone (23726-93-4) characterized as 'floral' and 'fruity' aromas were also present in winter wines, mainly at 30 months of ageing. Norisoprenoids, such as $\beta$-damascenone, are formed by an enzymatic reaction of carotenoids that are further subjected to catalytic reactions during wine ageing (Brillante et al., 2018).

Wine health benefits have been attributed to antioxidant, anti-inflammatory, anticarcinogenic, and antibacterial properties of sesquiterpenes (Siebert et al., 2008). Sesquiterpenes $\alpha$-Muurolene (10208-80-7) and $\alpha$-calacorene (21391-99-1), which contributes to woody, floral, and herbal aromas, were found mainly after 30 months of ageing.

\section{Acknowledgements}

Authors thank the Minas Gerais State Agency for Research and Development (FAPEMIG) and the Brazilian National Council for Scientific and Technological Development (CNPq) for funding, the São Paulo Research Foundation (FAPESP) for the scholarship and the southeast winter wine viticulturists for providing the grapes. 


\section{Authors' Contributions}

Conceptualization: Mota, R.V.; Souza, C.R.; Peregrino, I.; Hassimotto, N.M.A. Data acquisition: Mota, R.V.; Peregrino, I.; Rivera, S.P.T.; Souza, A.L. Data analysis: Mota, R.V.; Rivera, S.P.T.; Hassimotto, N.M.A. Design of methodology: Mota, R.V.; Peregrino, I.; Hassimotto, N.M.A. Writing and editing: Mota, R.V.; Peregrino, I.; Souza, C.R.; Hassimotto, N.M.A.

\section{References}

Amerine, M.A.; Ough, C.S. 1980. Methods for Analysis of Musts and Wines. John Wiley, New York, NY, USA.

Antalick, G.; Šuklje, K.; Blackman, J.W.; Meeks, C.; Deloire, A.; Schmidtke, L.M. 2015. Influence of grape composition on red wine ester profile: comparison between Cabernet Sauvignon and Shiraz cultivars from Australian warm climate. Journal of Agricultural and Food Chemistry 63: 4664-4672.

Belda, I.; Ruiz, J.; Esteban-Fernández, A.; Navascués, E.; Marquina, D.; Santos, A.; Moreno-Arribas, M.V. 2017. Microbial contribution to wine aroma and its intended use for wine quality improvement. Molecules 22: 189. doi: https://doi. org/10.3390/molecules22020189

Brillante, L.; Martínez-Lüscher, J.; Kaan Kurtural, S. 2018. Applied water and mechanical canopy management affect berry and wine phenolic and aroma composition of grapevine (Vitis vinifera L., cv. Syrah) in central California. Scientia Horticulturae 227: 261-271.

Cáceres-Mella, A.; Ribalta-Pizarro, C.; Villalobos-González, L.; Cuneo, I.F.; Pastenes, C. 2018. Controlled water deficit modifies the phenolic composition and sensory properties in Cabernet Sauvignon wines. Scientia Horticulturae 237: 105-111.

Cheynier, V.; Dueñas-Paton, M.; Salas, E.; Maury, C.; Souquet, J-M.; Sarni-Manchado, P.; Fulcrand, H. 2006. Structure and properties of wine pigments and tannins. American Journal of Enology and Viticulture 57: 298-305.

Cincotta, F.; Verzera, A.; Tripodi, G.; Condurso, C. 2015. Determination of sesquiterpenes in wines by HS-SPME coupled with GC-MS. Chromatography 2: 410-421.

Condurso, C.; Cincotta, F.; Tripodi, G.; Sparacio, A.; Giglio, D.M.L.; Sparla, S.; Verzera, A. 2016. Effects of cluster thinning on wine quality of Syrah cultivar (Vitis vinifera L.). European Food Research and Technology 242: 1719-1726.

Fabani, M.P.; Ravera, M.J.A.; Wunderlin, D.A. 2013. Markers of typical red wine varieties from the Valley of Tulum (San JuanArgentina) based on VOCs profile and chemometrics. Food Chemistry 141: 1055-1062.

Favero, A.C.; Amorim, D.A.; Mota, R.V.; Soares, A.M.; Souza, C.R.; Regina, M.A. 2011. Double-pruning of 'Syrah' grapevines: a management strategy to harvest wine grapes during the winter in the Brazilian Southeast. Vitis 50: 151-158.

Giusti, M.M.; Wrosltad, R.E. 2000. Characterization and Measurement of Anthocyanins by Uv-Visible Spectroscopy: Current Protocols in Food Analytical Chemistry. John Willey, New York, NY, USA.
Gürbüz, O.; Rouseff, J.M.; Rouseff, R.L. 2006. Comparison of aroma volatiles in commercial Merlot and Cabernet Sauvignon wines using gas chromatography-olfactometry and gas chromatography-mass spectrometry. Journal of Agricultural and Food Chemistry 54: 3990-3996.

Harbertson, J.F.; Spayd, S. 2006. Measuring phenolics in the winery. American Journal of Enology and Viticulture 57: 280288.

He, F.; Liang, N.; Mu, L.; Pan, Q-H.; Wang, J.; Reeves, M.J.; Duan, C-Q. 2012. Anthocyanins and their variation in red wines. II. Anthocyanin derived pigments and their color evolution. Molecules 17: 1483-1519.

Hunter, J.J.; Volschenk, C.G. 2018. Chemical composition and sensory properties of non-wooded and wooded Shiraz (Vitis vinifera L.) wine as affected by vineyard row orientation and grape ripeness level. Journal of the Science of Food and Agriculture 98: 2689-2704.

Loscos, N.; Hernández-Orte, P.; Cacho, J.; Ferreira, V. 2010. Evolution of the aroma composition of wines supplemented with grape flavor precursors from different varietals during accelerated wine ageing. Food Chemistry 120: 205-216.

Mota, R.V.; Amorim, D.A.; Fávero, A.C.; Gloria, M.B.A.; Regina, M.A. 2009. Physico-chemical characterization and bioactive amines in Syrah wines. I. Influence of growing season. Ciência e Tecnologia de Alimentos 29: 380-385 (in Portuguese, with abstract in English).

Mota, R.V.; Favero, A.C.; Silva, C.P.C.; Purgatto, E.; Shiga, T.M.; Regina, M.A. 2011. Wine grape quality of grapevines grown in the cerrado ecoregion of Brazil. Journal International des Sciences de la Vigne et du Vin 45: 101-109.

Mota, R.V.; Silva, C.P.C.; Favero, A.C.; Purgatto, E.; Shiga, T.M.; Regina, M.A. 2010. Physico-chemical composition of wine grapes berries in summer and winter growing seasons. Revista Brasileira de Fruticultura 32: 1127-1137 (in Portuguese, with abstract in English).

Ribéreau-Gayon, P.; Glories, Y.; Maujean, A.; Dubourdieu, D. 2006. Phenolic compounds. p. 141-204. In: Ribéreau-Gayon, P.; Glories, Y.; Maujean, A.; Dubourdieu, D., eds. Handbook of enology 2. The chemistry of wine: stabilization and treatments. John Wiley, New York, NY, USA.

Salettes, S. 2016. Minas Gerais (Brazil) wine in the country of coffee. = Minas Gerais (Brésil): du vin au pays du café. La Revue du Vin de France 599: 50-53 (in French).

Siebert, T.E.; Wood, C.; Elsey, G.M.; Pollnitz, A.P. 2008. Determination of Rotundone, the pepper aroma impact compound, in grapes and wine. Journal of Agriculture and Food Chemistry 56: 3745-3748.

Vidal, S.; Segurel, M. 2005. Knowledge of aromas of Grenache and Syrah = Connaissance des arômes du Grenache et de La Syrah. Guide de la Vinification Rhodanienne 9: 44-46 (in French). 